\title{
Understanding of the Shear Bands in Amorphous Metals
}

\author{
Eun Soo Park*
}

Department of Materials Science and Engineering, Seoul National University, Seoul 151-744, Korea

*Correspondence to:

Park ES,

Tel: +82-2-880-7221

Fax: +82-2-883-8197

E-mail: espark@snu.ac.kr

Received June 23, 2015

Revised June 24, 2015

Accepted June 24, 2015
Shear banding is an evidence of plastic instability that localizes large shear strains in a relatively thin band when a material is plastically deformed. Shear bands have attracted much attention in amorphous metals, because shear bands are the key feature that controls the plastic deformation process. In this article, we review recent advances in understanding of the shear bands in amorphous metals regarding: dislocations versus shear bands, the formation of shear bands, hot versus cold shear bands, and property manipulation by shear band engineering. Although there are many key issues that remain puzzling, the understanding built-up from these approaches will provide a new insight for tailoring shear bands in amorphous metals, which potentially leads to unique property changes as well as improved mechanical properties. Indeed, this effort might open a new era to the future use of amorphous metals as a new menu of engineering materials.

Key Words: Amorphous metal, Plastic deformation, Shear band, Shear band engineering, Property manipulation

\section{DISLOCATIONS VERSUS SHEAR BANDS}

Amorphous metals, especially bulk metallic glasses (BMGs), are highly applicable for new structural materials as a substitute for conventional crystalline metals due to superior mechanical properties, and thus occupy a unique niche compared with other classes of engineering materials. However, they usually suffer from a strong tendency for shear localization and macroscopically brittle failure at room temperature, which restricts structural application of BMGs (Schuh et al., 2007). Although the underlying deformation physics of these materials establishes less firmly as compared with crystalline metals, understanding of shear banding is particularly important in amorphous metals because shear bands play a crucial role in controlling plasticity and failure at ambient temperature. First of all, to discuss why the deformation behavior of amorphous metals presents a great contrast to crystalline metals, the atomistic understanding of these two materials is necessary.

It has been widely known in soil mechanics that the shear of randomly closed-packed grains causes dilatation phenomena (Reynolds, 1885), e.g., the disappearance of water underneath feet on a wet beach because of the shear induced by the weight. The same mechanism can be applied to the atomic scale deformation in amorphous metals. Fig. 1 shows the atomic structures of crystalline and amorphous metals. When the crystalline metals are exposed to shear stress, they maintain the initial volume during plastic deformation because the periodicity along the slip planes provides identical atomic positions for the sheared material. On the other hand, amorphous metals, which have a random structure, increase their internal free volume as they plastically deform and must leave some voids. In general, the nucleation of nano-voids is more favorable on tensile side than compressive side, which indicates that the hydrostatic component of stress affects void nucleation. As a result of deformation, dislocations and shear bands are formed in crystalline and amorphous metals, respectively (Fig. 2) (http://faculty.virginia.edu/teamhowe/ files/EMFacility.html), which are caused by different micromechanisms. Crystalline metals use the dislocation motions

This work was supported by the National Research Foundation of Korea (NRF) grant funded by the Korean government (Ministry of Science, ICT and Future Planning) (No. 2012R1A2A2A01046729). The author also benefited from the Center for Iron and Steel Research (RIAM) and Engineering Research Institute at Seoul National University.

@ This is an open-access article distributed under the terms of the Creative Commons Attribution Non-Commercial License (http://creativecommons.org/licenses/by-nc/4.0) which permits unrestricted noncommercial use, distribution, and reproduction in any medium, provided the original work is properly cited.

Copyrights (C) 2015 by Korean Society of Microscopy 


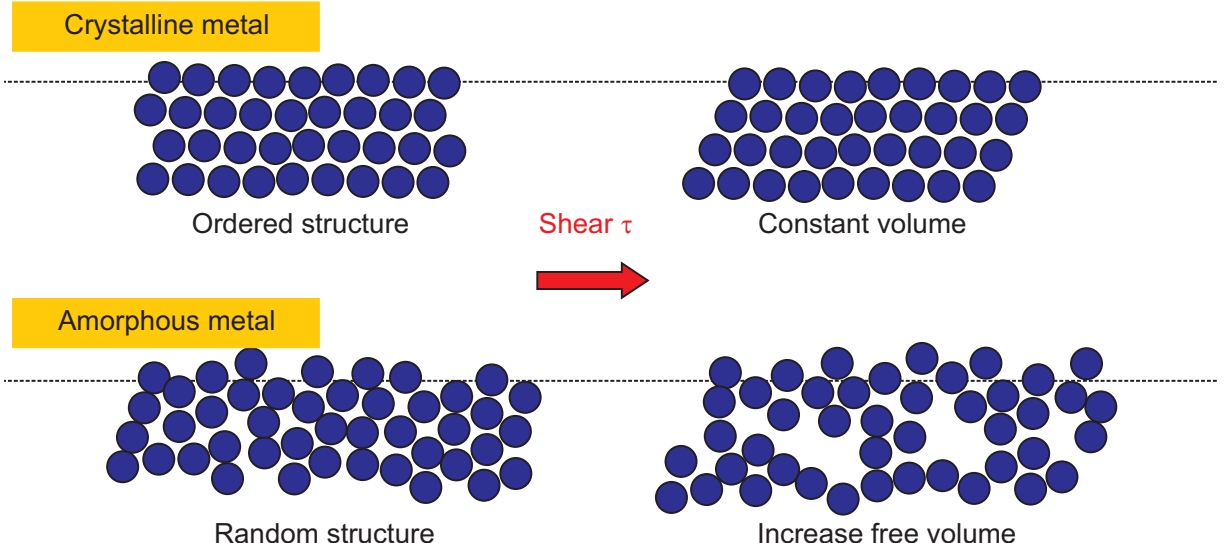

Fig. 1. Atomic structures of crystalline and amorphous metal.
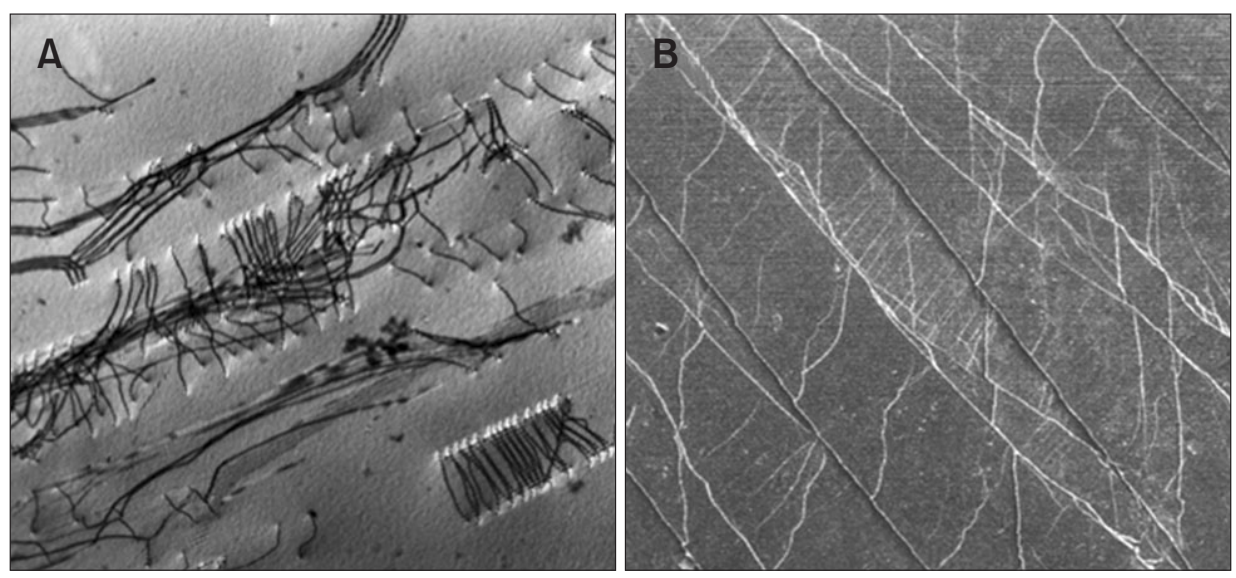

Fig. 2. Dislocations versus Shear bands. (A) Transmission electron microscope image of deformed 316 stainless steel showing dislocations and dipoles on (111) planes (http://faculty.virginia.edu/ teamhowe/files/EMFacility.html). (B) Scanning electron microscope image of deformed bulk metallic glass showing multiple shear bands (=shear steps) on the surface of the sample. that consist of incrementally breaking bonds, and the dislocations move along preferred crystallographic planes and directions, which enable the material to perform work hardening behavior. However, in single phase amorphous metals, the plastic flow is initiated in some locally perturbed regions and localizes due to strain softening through shear bands by accumulation of flow defects, which is known from observation of atomic-scale voids by position annihilation and electron microscopy (Fig. 3) (Gu et al., 2003; Schuh et al., 2007). Thus, it is difficult to disturb the tendency for abrupt fracture along the planes of those shear bands, especially under tensile loading, which is a strong deterrent to using amorphous metals in structural applications. The present work aims to provide a focused up-to-date review on shear bands in amorphous metals with a specific emphasis upon fundamentals, deformation mechanisms and manipulations, which can offer insights on important questions regarding how to achieve ductilization of amorphous metals.

\section{FORMATION OF SHEAR BANDS}

The exact nature of local atomic motion in deforming amorphous metals is not completely understood, although there is extensive consensus that the fundamental unit procedure underlying deformation must be a local rearrangement of atoms that can accommodate shear strain. The structural rearrangement is critical in controlling resistance to flow and is central to the evolution of many properties of amorphous metals. Fig. 4 shows two-dimensional schematics of the atomistic deformation mechanisms proposed for amorphous metals, including a local atomic jump (Fig. 4A; Spaepen, 1997) and a shear transformation zone (STZ) (referred to as a "flow defect", " $\tau$ defect" or "local inelastic transition”) (Fig. 4B; Argon, 1979).

\section{Free Volume Theory}

The total volume of an amorphous metal can be separated into the space occupied by the dense atomic clusters and the empty space among these atomic clusters due to packing frustrations. The empty space, termed the free volume, around constituent atoms has a lower atomic coordination than those in the densely packed reference atomic clusters. In the free-volume regions, where mechanical coupling to the surrounding is weak, inelastic relaxation becomes possible by local 

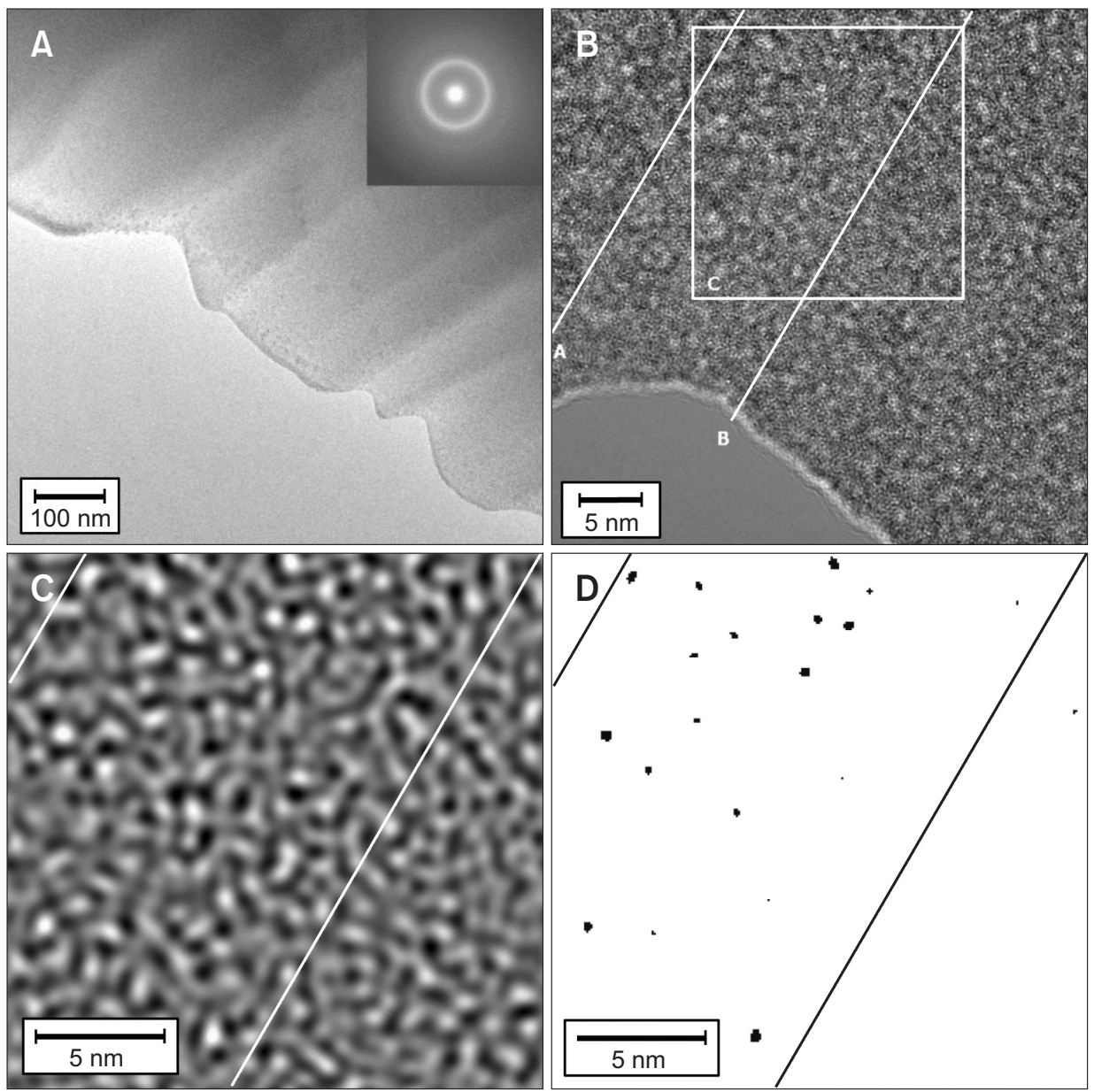

Fig. 3. (A) Low magnification and (B) high resolution transmission electron microscope micrograph (large defocus value, $\Delta \mathrm{f}=-260 \mathrm{~nm}$ ) taken from the widely spaced shear band area produced by bending $\mathrm{Zr}_{52.5} \mathrm{Ti}_{17.9} \mathrm{Cu}_{20} \mathrm{Ni}_{8} \mathrm{Al}_{10}$ amorphous metal. (C) Fourier filtered image of the square ' $C$ ' in Fig. 3B, (D) same image as Fig. 3C with a threshold chosen to reveal features with brightness about 3.0 standard deviations greater than the mean. Contrast of image in Fig. 3D was reversed so that the black spots in the image correspond to bright features (regions of excess free volume) in image Fig. 3C. In Fig. 3B-D, it is clear that free volume has accumulated preferentially in the shear band. Additionally nanoscale voids of $\sim 1 \mathrm{~nm}$ size and less are observed within the shear band in Fig. 3D. Reused from the articles of Schuh et al. (2007) (Acta Mater. 55, 4067-4109) and Gu et al. (2003) (Mater. Res. Soc. Proc. 754, CC7.9.1.-CC7.9.6.) with permission through "Copyright Clearance Center". atom rearrangements, without affecting the surroundings significantly. Thus, these sites (recently, called soft spots; Ding et al., 2014) may be the preferred regions at which to initiate the amorphous structure destabilization caused by either temperature (glass transition) or applied stresses (local shearing).

An important feature of the plastic deformation of amorphous metals is deformation-induced strain softening by mechanical dilatation. Shear deformation by sliding at high stress leaves behind geometric defects with a low packing density of amorphous metals. The stress-driven-defect creation procedure is a function of the instantaneous stress, temperature, and defect concentration. Making defects requires the creation of excess volumes, which is similar to granular materials as explained before.

On the basis of this idea, Spaepen (1977) developed a singleatom model to describe the formation of free volumes through the elastic energy consideration of squeezing an atom into a small empty space (Fig. 4A). Macroscopic plastic flow is assumed to occur as sum of individual atomic jumps. In order for an atomic to jump, there must be a hole large enough to accommodate volume like diffusion mechanism.
When an external stress is applied, however, energy barrier is tilted, and direction of atomic jumps moves forward to stress direction. This flow can describe the general case of plastic flow in amorphous metals. At low temperatures, at which the diffusive decay of such activation dilatation caused by the mechanical deformation may be very slow, the shear regions can retain a large non-equilibrium component of shear-induced excess free volumes, which leads to lowering of the deformation resistance, strain softening, and thereby strong shear localization. However, recent observations on three dimensional colloidal glass (Schall et al., 2007) have implications for the free volume theory, which needs to be extended from the original single atomic jumps to multiatom rearrangements.

\section{Shear Transformation Zone (STZ) Theory}

Although the free volume model provides a simple and clear explanation for the strain softening, free-volume site initiation cannot stand for the deformation process itself. This model is not enough to clarify the motion and rearrangement of constituent atoms within shear bands during plastic flow. Therefore, STZ model was proposed by Argon (1979) and 
Argon and Kuo (1979) on the basis of an atomic-analog bubble-raft model, which explains the shear deformation as spontaneous and cooperative reorganization of a small cluster of randomly close-packed atoms (Fig. 4B). The STZ is essentially a local cluster of atoms (between a few and perhaps $\sim 100$ atoms) that undergoes an inelastic shear distortion from one relatively low energy configuration to another activated configuration of higher energy and volume, which leads to stress and strain redistribution around the STZ region. According to this model, the local motion of STZs around free-volume sites can push apart surrounding atoms along activation paths, resulting in a dilatation and strain softening (Schuh et al., 2007).

\section{Deformation Map of Amorphous Metals}

The deformation map developed by Ashby and Frost for crystalline metals (Ashby, 1972) is a convenient guideline for surveying the various modes of plastic deformation of materials. The deformation of amorphous metals is shared by both the free volume theory and STZ theory viewpoints. In contrast with the deformation of crystalline metals, only a single mechanism is required to explain the basic features of deformation of amorphous metals over the full range of temperatures, strain rates and possible glass structure. This mechanism, whether one subscribes to a free volume theory or STZ theory, may occur homogeneously throughout an amorphous body, or may occur in a localized region during the formation of a shear band. Although the macroscopic mechanical response may differ between these two cases, the

\section{A}

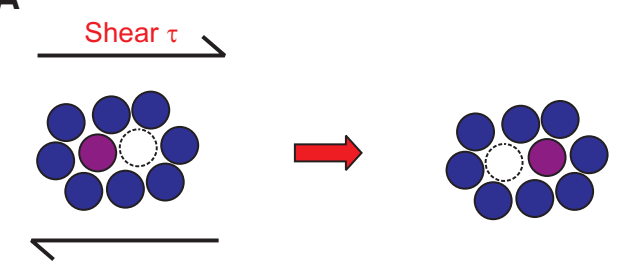

B

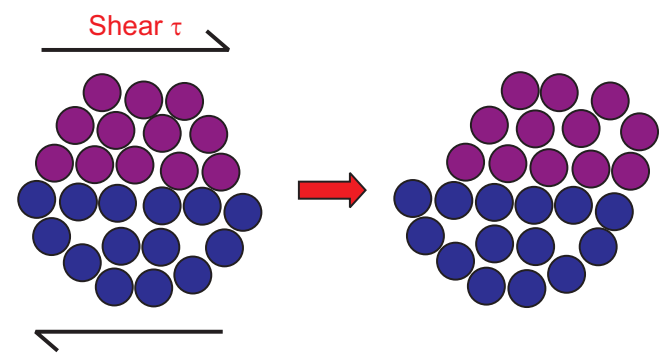

Fig. 4. Two-dimensional schematic diagram of the atomistic deformation mechanisms proposed for amorphous metals, including an individual atomic jump (macroscopic diffusion and flow) (A, Spaepen [1977]) and a shear transformation zone (spontaneous and cooperative reorganization) (B, Argon [1979]). deformation is fundamentally the same. Fig. 5 is a schematic deformation map of amorphous metals based on empirical information constructed by Spaepen (1977), which indicates various modes of deformation. There are two basic modes of deformation: homogeneous flow, where each volume element of the specimen contributes to the strain, and inhomogeneous flow, where the strain is concentrated in a few very thin shear bands.

Homogeneous flow occurs at low stresses and high temperatures, which normally occurs above and near the glass transition temperature. In this deformation regime, the flow is close to Newtonian viscous flow, as shown in the spacing of the strain rate contours on the map. In uniaxial tension, specimen's each volume element undergoes the same strain, and finally thins down uniformly during deformation. Fracture occurs after extensive plastic flow, when some section of the specimen has narrowed down to zero thickness. The general flow equation can be derived in terms of diffusion-like mechanism, which is based on a dynamic equilibrium between shear-induced disordering process (creation of free volume) and diffusion controlled reordering process (annihilation of free volume) at steady state. In this mechanism, the free volume of an atom is described as the part of its nearest neighbor position where the atom can move around without

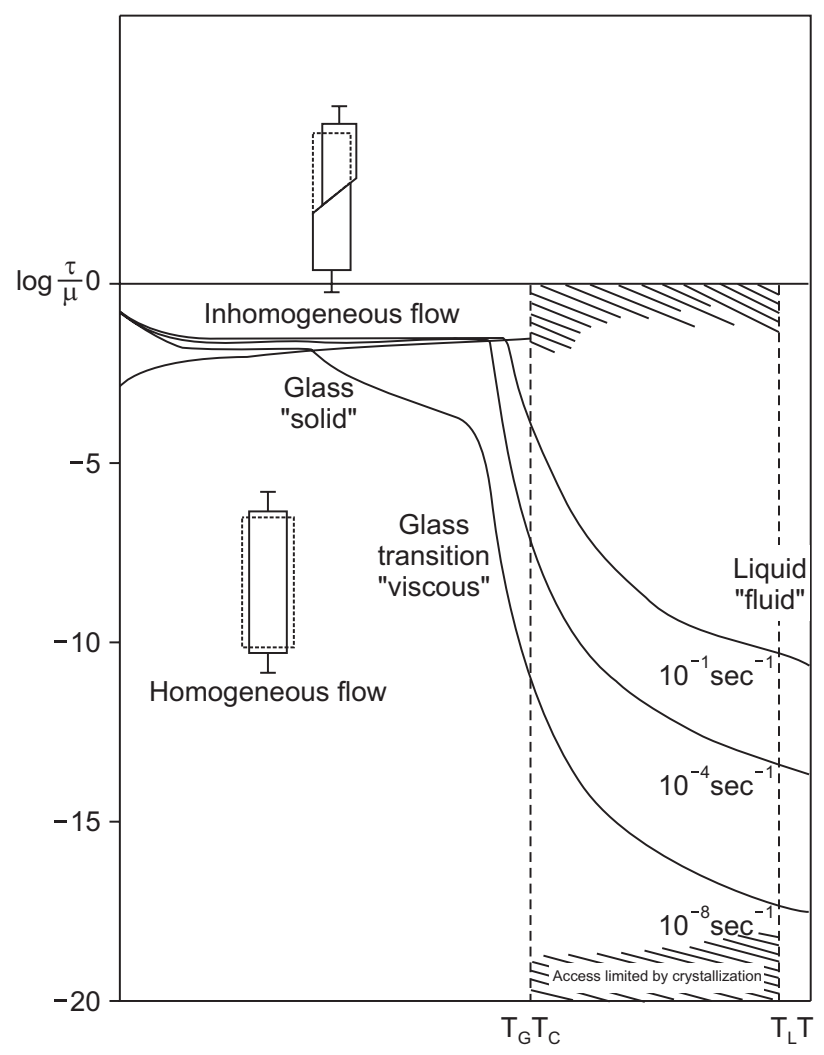

Fig. 5. A deformation mechanism map (steady-state strain rate as a function stress and temperature) for a typical amorphous metal. Reused from the article of Spaepen (1977) (Acta Mater. 25, 407-415) with permission through "Copyright Clearance Center". 
an energy change.

On the other side of the map, inhomogeneous flow occurs at different conditions - high stress levels and relatively low temperatures. In this regime, the stress is very strain rate insensitive, so that the flow approaches ideally plastic behavior. The flow stress for a given strain rate, when normalized by the temperature dependent shear modulus, is almost constant with temperature, except at a low temperature and near glass transition temperature. On the deformation map, this results in very closely spaced, almost horizontal strain rate contours. The basic physical process underlying this inhomogeneous flow phenomenon is a local softening of the material in the shear bands.

Although the original deformation map of Spaepen (1977) has been revisited by later authors (Argon \& Kuo, 1979; Megusar et al., 1979; Lu et al., 2003), recently Schuh et al. $(2004,2007)$ reconstructed the map and updated a number of recent observations in amorphous metal deformation. They develop two complementary maps; one in coordinates of stress and temperature (Fig. 6A), which follows the form originally proposed by Spaepen (1977) and a second in strain rate-temperature space (Fig. 6B), which was delineated by Megusar et al. (1979). When stress is on the abscissa of the map, strain rate can be represented as a series of contours; conversely, contours of stress lie in the strain rate-temperature map. In both maps, they present stresses as fractions of the shear modulus, which allows for at least approximate generalization to many amorphous metals. The main division on the map separates homogeneous deformation at high temperatures and low stresses/rates from inhomogeneous flow (shear localization) at lower temperatures and higher stresses/rates. In the homogeneous regime contours for steady-state flow are indicated, as is the transition from Newtonian to non-Newtonian flow. In the inhomogeneous regime, the effect of confining hydrostatic pressure is shown, and various degrees of flow serration are denoted in Fig. 6B (Schuh et al., 2007).

The deformation maps are a simple but informative view of amorphous metal deformation as a function of strain rate, temperature and stress. They may be used as semiquantitative tools, e.g., for rationalizing observed trends with applied strain rate or temperature, or for comparing observed mechanical responses for different amorphous metals tested at a room temperature but at different homologous temperatures $\left(\mathrm{T} / \mathrm{T}_{\mathrm{g}}\right)$.

\section{Shear Band Nucleation and Propagation in Amorphous Metals}

The plastic deformation, i.e., irreversible shear, of amorphous metals is essentially a biased accumulation of local strains incurred through the redistribution of free volume and the operation of STZ, which can develop the nucleation and propagation of shear bands. Shear band behavior can be described using the excess volume concept used in above two models. When the initial amorphous structure is exposed to elastic loading, the isotropic dilatation takes place and the free volume increases. When the stress reaches a critical value, yielding occurs to give macroscopically observable permanent shape change, often immediately followed by the initiation of a shear band. Thus, structural inhomogeneities and defects that introduce stress concentrations will naturally tend to promote shear band initiation. The nucleation of a shear band is the connection of these shear-induced free volumes, which causes brighter contrast than matrix in transmission electron
A

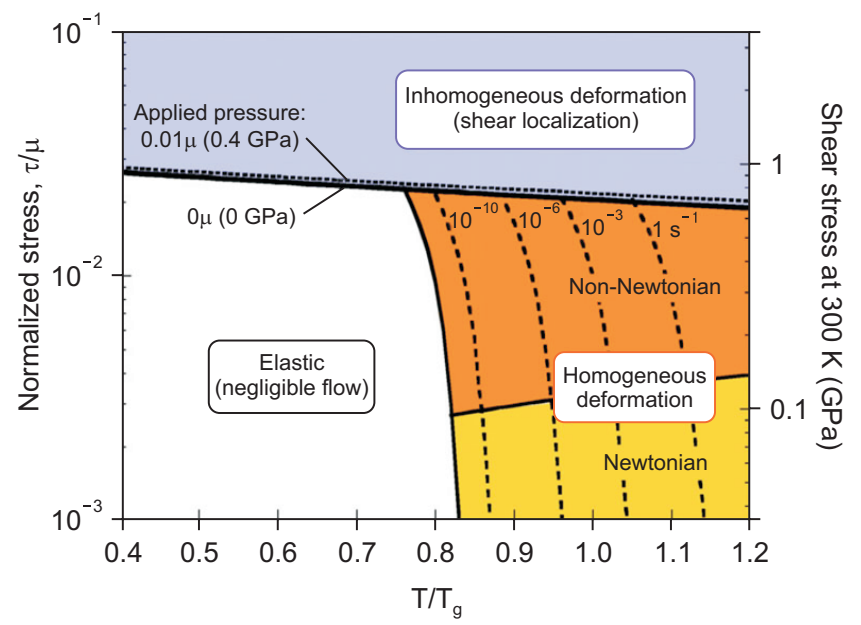

B

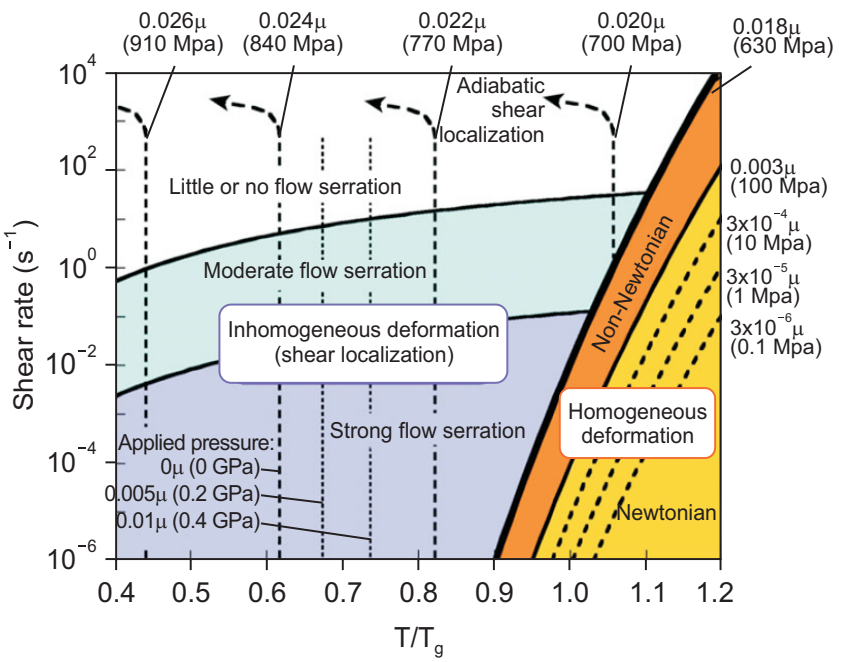

Fig. 6. Deformation map for amorphous metals in stress-temperature (A) and strain rate-temperature (B) axes. Reused from the article of Schuh et al. (2007) (Acta Mater. 55, 4067-4109) with permission through "Copyright Clearance Center". 


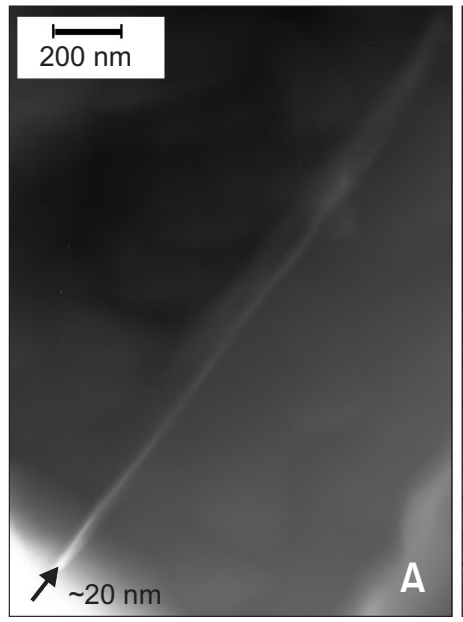

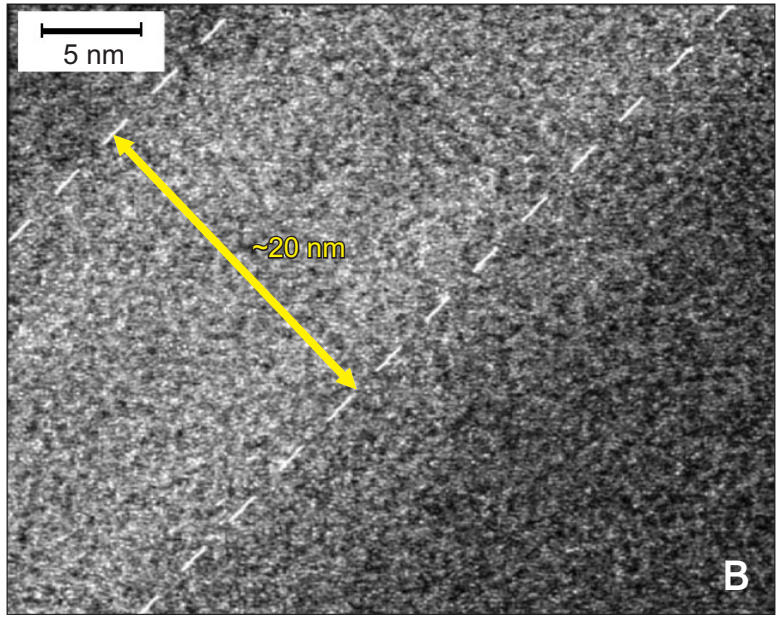

Fig. 7. Low magnification bright field transmission electron microscope (TEM) micrograph (A) and high resolution TEM micrograph (B) of shear band formed during bend test of $\mathrm{Fe}_{79.3} \mathrm{~B}_{16.4} \mathrm{Si}_{4} \mathrm{C}_{0.3}$ ribbon (thickness: $\sim 40 \mu \mathrm{m}$ ). microscope observation as shown in Fig. 7. Actually, the shear band is not one-dimensional line but three-dimensional spaces with around 10 100 nm thickness in an amorphous metal, which is quite small in comparison with adiabatic shear bands (typically 10 100 $\mu \mathrm{m}$ thickness) in crystalline metals. If the stress is still applied after shear band formation, the plastic strain localized into these shear bands. And this elastic energy raises local temperature inside shear bands, eventually leading to the shear softening-decrease in the local viscosity. The repeated localization of shear strain procedure results in the catastrophic failure, which is same as an abrupt propagation of a shear band. To improve the plasticity of amorphous metals, multiple shear bands should be nucleated for even distribution of strain, which hinders the propagation motion of shear bands. This phenomenon can be achieved in composites with different scale heterogeneities and phases, where the microstructure can be tailored with the goal of achieving a high density of shear bands or disturbing shear band propagation.

\section{HOT VERSUS COLD SHEAR BANDS}

There has been considerable debate as to whether shear localization in amorphous metals is mainly due to thermal effects or shear-induced disordering such as dilatation. Recent studies have proposed that shear bands in amorphous metals can operate 'hot' (with significant local heating), or 'cold' (i.e., at essentially the temperature of the bulk material). The temperature in the bands during their operation is of key importance to understand this localized mode of deformation.

\section{Hot Shear Bands}

Compared with conventional engineering alloys, amorphous metals exhibit lower values of Young's modulus (up to 30\%) but a higher value of elastic strain limit (about 2\%) (Ashby \&
Greer, 2006; Cheng \& Ma, 2009). Besides, these values mean that amorphous metals can store an elastic strain energy per unit volume (up to some $0.5 \mathrm{MJ}^{-3}$ ) more than any other crystalline metal. And high loads at yield imply that significant elastic energy may also be stored in the loading systems. Moreover, when yielding starts, the possible dissipation of these energies is not throughout the entire volume, but in the very small volume fraction of material within the shear bands. Thus, it is clear that there is the potential for substantial temperature rises (similar to adiabatic shear bands in crystalline metals). Indeed, it should come as no surprise that the fracture of amorphous metals is often accompanied by sparking, the formation of liquid droplets and the ejection of particles (heated by several hundred degrees) (Bruck et al., 1996; Liu et al., 1998; Gibert et al., 1999).

The volumes in shear bands being so small and such a small fraction of the sample volume, and the times of band operation being so short, it is difficult to explore the structure and properties of the material in a shear band during its operation. Although there are a few indirect observations to capture a snapshot of the thermal profile around a shear band (Lewandowski \& Greer, 2006), the most obvious approach is to use a direct technique such as infra-red thermography. Interestingly, this can image the hot zone around shear bands and can detect shear-banding on progressive loading well before ultimate sample failure as shown in Fig. 8 (Yang et al., 2004).

\section{Cold Shear Bands}

Alternatively, in stable plastic deformation such heating is far from unavoidable, and the question Spaepen (2006) raised "Must shear bands be hot?" Still remains of considerable interest. Recently, it was reported that in a displacementcontrolled compression test of a BMG, the dominant shear band can slide in a stick-slip manner, giving rise to controlled and sustained plastic strain. The analysis predicts an acceleration-deceleration cycle that explains the stop- 
A

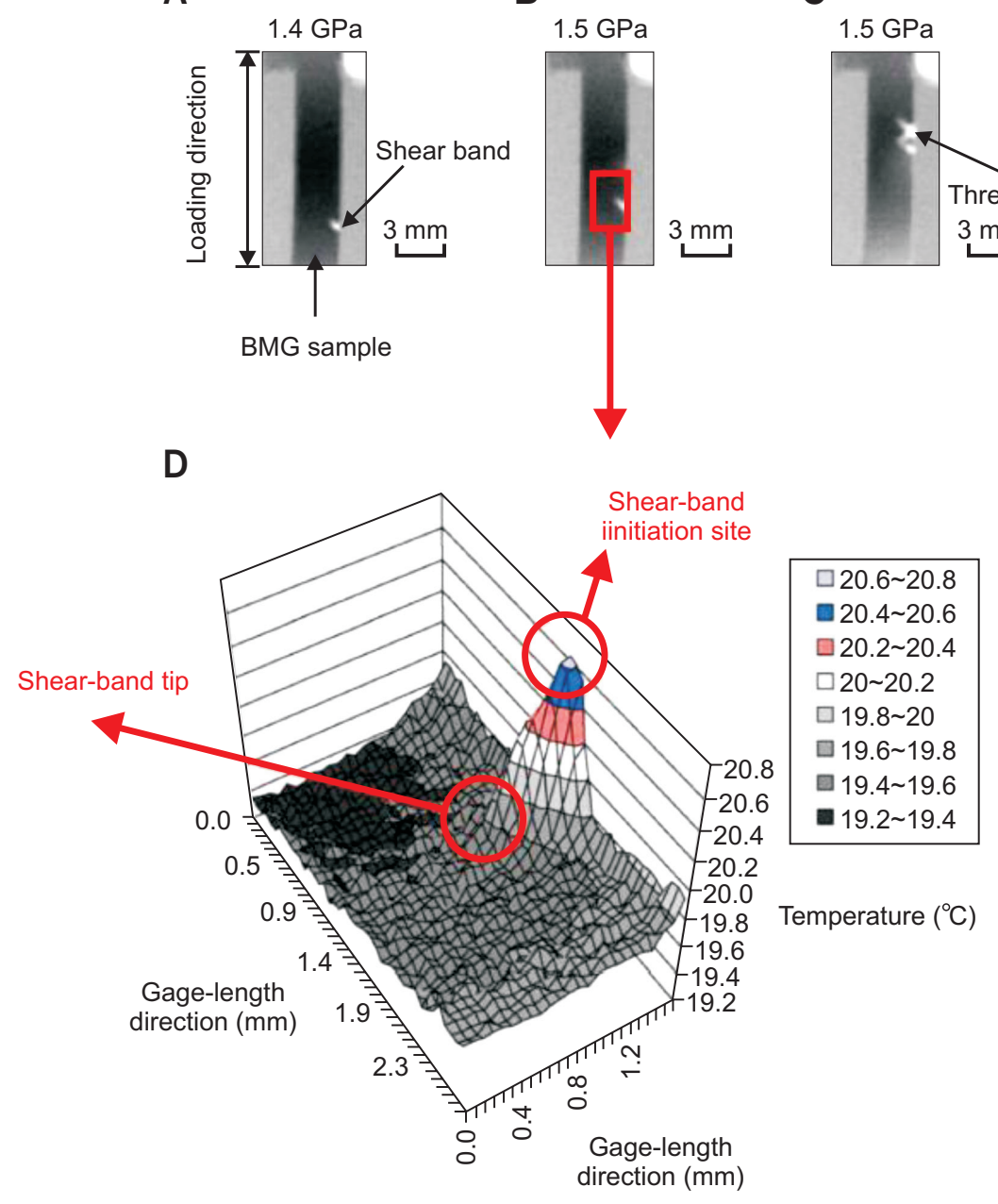

B
Fig. 8. Infra-red thermography (400 frames per second) used to observe shear bands in a $\mathrm{Zr}_{52.5} \mathrm{Cu}_{17.9} \mathrm{Ni}_{14.6} \mathrm{Al}_{10.0} \mathrm{Ti}_{5.0}$ bulk metallic glasse sample during tensile testing. Reused from the article of Yang et al. (2004) (Intermetallics 2, 1265-1274) with permission through "Copyright Clearance Center". and-go behavior observed in experiments. The temperature rises in such shear bands are predicted to be very small, quantitatively illustrating that such shear bands can be cold (Cheng et al., 2009). The cold shear banding provides an explanation for the "large plasticity" observed in some BMGs, especially in small (around $1 \mathrm{~mm}$ ) samples and in submicron pillars (Guo et al., 2007; Shan et al., 2008). In these cases, it is experimentally observed that the shear band exhibits the stick-and-slip behavior, low speed of the shear offset growth, and striations on the sheared surfaces (Song et al., 2008; Song \& Nieh, 2009). Of course, multiple shear bands do often come into play in BMGs that can accommodate large plastic strains. However, as the hot shear bands directly develop in a runaway instability (catastrophe), the controlled and serrated sliding of the major shear band is also an important condition for multiple shear bands to have the opportunity to contribute to the plastic deformation. Indeed, the discussion regarding the role of cold shear banding should have impact on the understanding of shear-band behavior of BMGs. Fig. 9 shows the transition from cold (stick-slip) to hot (runaway) shear

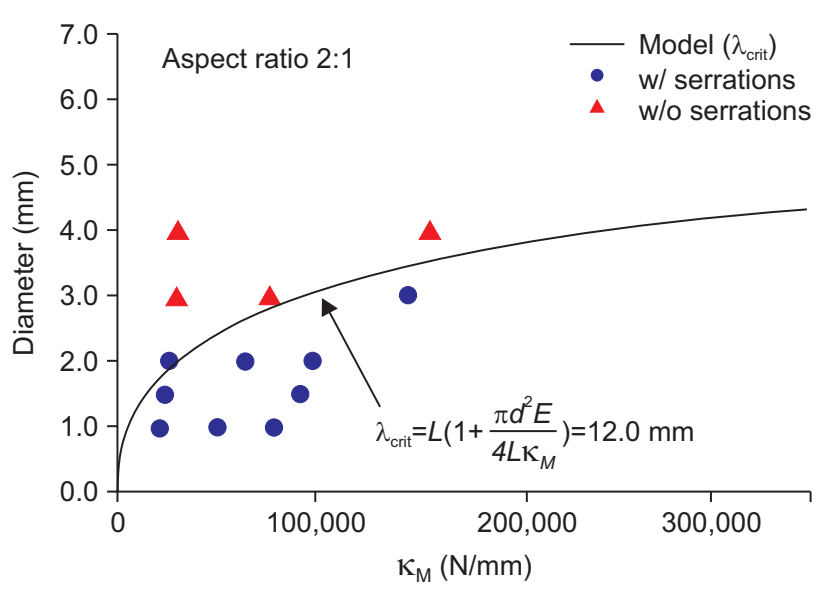

Fig. 9. The predicted functional form of $\lambda_{\text {crit }}$ separates two regimes (stick slip versus runaway instability). For the $\mathrm{Zr}$-based amorphous metal, this critical value is $12 \mathrm{~mm}$ (or diameter of $6 \mathrm{~mm}$, for an aspect ratio of 2). Here, $d$ denotes the sample diameter and $\kappa_{M}$ denotes the machine stiffness. Two different sample-aspect ratios exhibit consistent $\lambda_{\text {crit }}$ indicating that $\lambda_{\text {crit }}$ is a material property related to intrinsic plasticity. Reused from the article of Cheng et al. (2009) (Phys. Rev. B 80, 134115) with permission through "Copyright Clearance Center". 
band depending on the instability index $\mathrm{L}(1+\mathrm{S})$, where $\mathrm{L}$ is the sample height and $\mathrm{S}$ is defined by $\mathrm{S}=\kappa_{S} / \kappa_{M}=\pi d^{2} E / 4 L \kappa_{M}$ in Han et al. (2009), with all other factors held constant. But the transition is often influenced by other aspects of the loading as well by the characteristics of the amorphous metal itself (Cheng et al., 2009). Understanding cold shear bands as well as hot shear bands provides physical insights into the shear instability processes and offers useful information for improving the plasticity of BMGs.

\section{Crystallization in Shear Bands}

It is well known that room-temperature mechanical deformation, especially under compressive mode, of amorphous metals with structural instability can induce crystallization as shown in Fig. 10 (Gu et al., 2003; Chang et al., 2006). The mechanism by which crystallization occurs in shear bands is the subject of considerable debate. Of course, amorphous metals crystallize during heating, and it has therefore seemed natural to associate crystallization in shear bands with localized heating. But, as in considering the origin of shear localization, the increased atomic mobility in shear bands can be a result of temperature rise or of structural change caused by shear (characterized, for example, by structural disorder and free-volume generation). Thus, many studies of deformation induced crystallization have compared it with thermal crystallization (i.e., induced by simple annealing) in an attempt to conclude whether it has its origin in heating or in free-volume generation. However, the clear origins of crystallization are still unsolved mystery. It should be noted that there is variety of deformation behavior, associated with the range from cold to hot shear bands. Indeed, we can expect a similar variation in the origins of crystallization. At this point we can explain further that the location of deformation-induced crystallization is significant. If a shear band is cold, crystallization occurs within it; if the band is hot, crystallization occurs outside it, and indeed the high temperatures at the center of the band would lead to reamorphization (Greer et al., 2013). The crystallization within shear bands may occur during or after the shear event, which may contribute to energy absorption, thereby hindering crack propagation and premature failure. When it occurs during shear it is associated with improvements in plasticity of amorphous metals (Pauly et al., 2010a, 2010b), which can exploit deformation-induced phase transformation to enhance plasticity (Hofmann, 2010; Wu et al., 2010; Wu et al., 2012). Indeed, it has been suggested that deformation of amorphous metals may be a way of generating unique nanostructures that is a useful alternative to simple thermal annealing.

\section{SHEAR BAND ENGINEERING}

The manipulation of microstructure to optimize properties (called tailor-made material design) forms a large part of the classical field of physical metallurgy. Crystalline metals exhibit many microstructural features such as precipitates, dislocation configurations, twin boundaries and grain boundaries, which can be controlled by optimizing the nature, population density and distribution. Although amorphous metals have no microstructure, it is required to consider how amorphous metals might be designed to optimize their properties by control of shear banding (Greer et al., 2013). For example, by cold rolling of amorphous metals, we can control the density of shear bands, which exhibit same composition and different free volume contents (occasionally, degree of crystallinity) with amorphous matrix. With increasing density
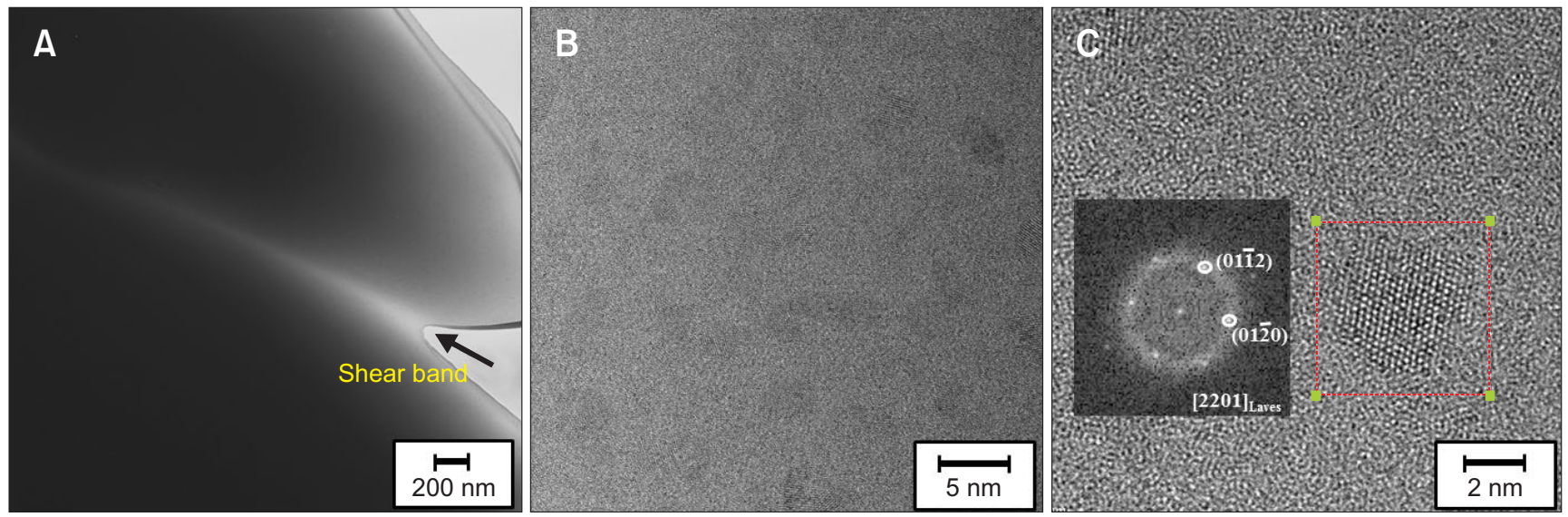

Fig. 10. (A) Low magnification bright field transmission electron microscope (TEM) micrograph showing a region containing the shear band after deformation of $\mathrm{Ti}_{40} \mathrm{Zr}_{29} \mathrm{Cu}_{9} \mathrm{Ni}_{8} \mathrm{Be}_{14}$ bulk metallic glasse sample. (B) High resolution TEM micrograph showing a part of the area indicating the existence of small crystallites. (C) High resolution TEM micrograph of an area containing one of the crystals (the inset shows the Fourier transformation of the region containing the particle). Reused from the article of Chang et al. (2006) (Scripta Mater. 55, 509-512) with permission through "Copyright Clearance Center". 
A

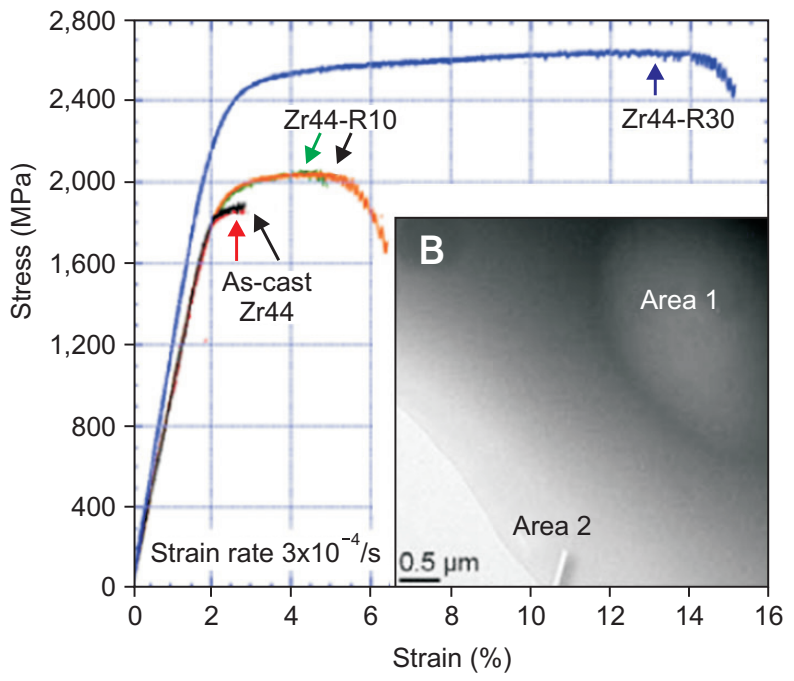

C

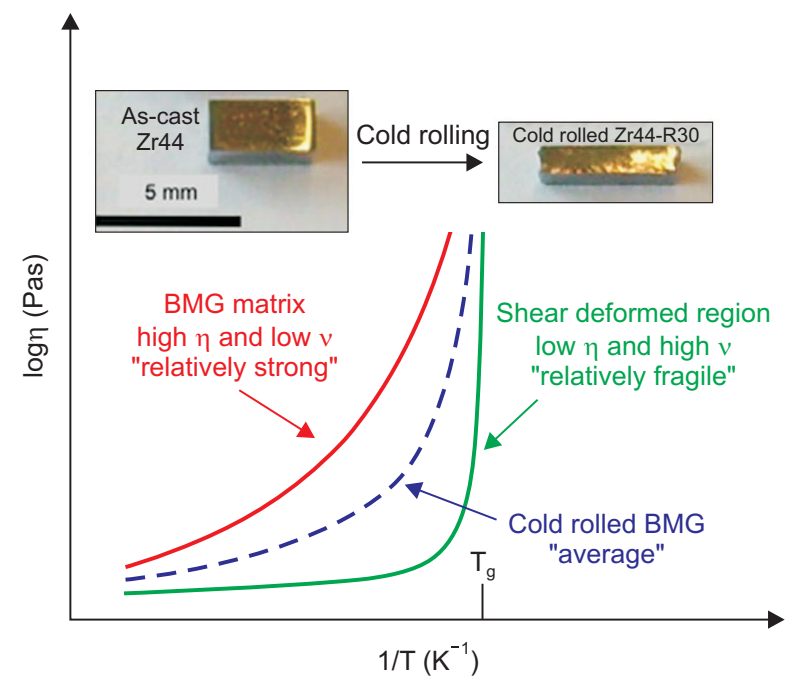

Fig. 11. (A) Compressive stress-strain plots of as-cast and differently cold rolled $\mathrm{Zr} 44\left(\mathrm{Zr}_{44} \mathrm{Ti}_{11} \mathrm{Cu}_{9.8} \mathrm{Ni}_{10.2} \mathrm{Be}_{25}\right)$, showing the improvement of intrinsic plasticity upon cold rolling of "brittle" BMGs at room temperature. (B) Transmission electron microscope bright field image of Zr44-R30. (C) Schematic illustration of transition in bulk metallic glasses from the strong to the fragile side of the Arrhenius plot upon cold rolling. Upper inset is outer look of as-cast and Zr44-R30 before compression test. Reused from the article of Lee et al. (2010) (Scripta Mater. 62, 678-681) with permission through "Copyright Clearance Center".

Tensile side
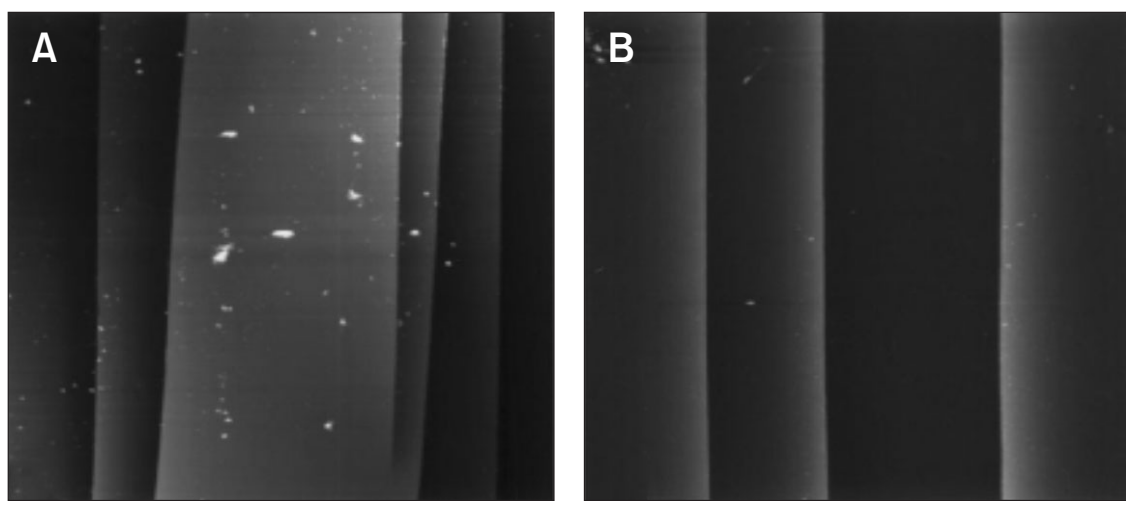

$100 \mathrm{~nm}$

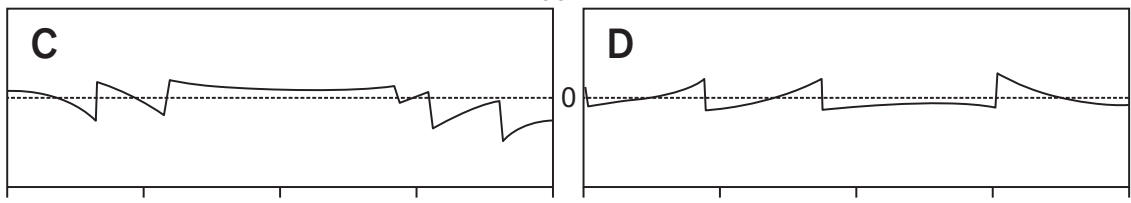

$-100 \mathrm{~nm}$

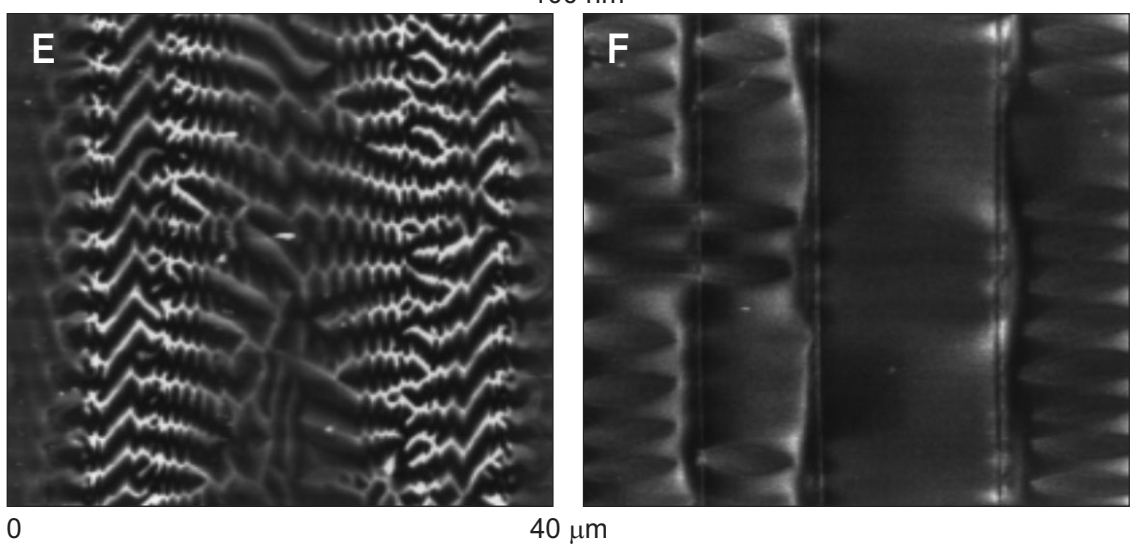

Fig. 12. Atomic force microscopy (AFM)/ Magnetic force microscopy (MFM) data of bent $\mathrm{Fe}_{79.3} \mathrm{~B}_{16.4} \mathrm{Si}_{4} \mathrm{C}_{0.3}$ amorphous ribbons for each side (tensile, compressive side). Topographic images of AFM in plain view of the tensile (A) and compressive (B) sides. Corresponding line scans (C, D) are shown for each to show the shear step profiles in better detail. At the bottom are the corresponding MFM images (E, F) of magnetic structures. Reused from the article of Brown et al. (1999) (J. Appl. Phys. 85, 4415) with permission through "Copyright Clearance Center". 
of shear bands, the first peak position in the X-ray diffraction pattern shifts slightly to the low angle side and the width of the peak at half height becomes larger, which is related to stabilization of the internal structure of the amorphous phase (low $\eta$ and high Poisson's ratio, $v$ ). Although Young's modulus, the fracture stress and the hardness are reduced after cold rolling, which should be observable softening effect (Masumoto \& Maddin, 1975) other mechanical properties including plasticity of BMG can be improved when its structure becomes more effectively inhomogeneous amorphous structure. Indeed, mechanical processing such as cold rolling, uniaxial compression, channel-die compression, shot-peening, and imprinting permits the development of shear-band patterns, in some cases with controlled directionality, which causes harder (denser) and softer regions in amorphous metals with attractive mechanical properties as shown in Fig. 11 (Lee et al., 2010). Besides mechanical property, effective mechanical processing of amorphous metals also leads to unique property changes such as processing-induced magnetic structure as shown in Fig. 12 (Brown et al., 1999), which can change directional variation of magnetic properties. Thus, it can be concluded that shearband engineering is possible to manipulate properties of amorphous metals without clear microstructural features and there are fruitful prospects for future improvements of the performance of amorphous metals, although it is not possible with shear-band engineering alone.

\section{SUMMARY AND OUTLOOK}

In this review, we have summarized our understanding about key issues of shear bands in amorphous metals: dislocations versus shear bands, the formation of shear bands, hot versus cold shear bands, and property manipulation by shear band engineering. But there still remain unsolved issues and important topics, such as STZ structure, the effect of environmental conditions on shear banding, strain continuity around shear bands, and particularly at shear-band intersections, the role of shear bands in fatigue cracking, mechanics predictions of shear bands and multilevel modeling of shear band behavior. The answers would undoubtedly lead to superior understanding and control of shear bands, potentially leading to unique property changes as well as improved mechanical properties in amorphous metals. Indeed, understanding of the shear bands holds the key to the future use of amorphous metals as a new class of structural materials.

\section{CONFLICT OF INTEREST}

No potential conflict of interest relevant to this article was reported.

\section{REFERENCES}

Argon A S (1979) Plastic deformation in metallic glasses. Acta Mater. 27, 47-58.

Argon A S and Kuo H Y (1979) Plastic flow in a disordered bubble raft (an analog of metallic glass). Mater. Sci. Eng. 39, 101-109.

Ashby M F (1972) A first report on deformation-mechanism maps. Acta Mater. 20, 887-897.

Ashby M F and Greer A L (2006) Metallic glasses as structural materials. Scripta Mater. 54, 321-326.

Brown G W, Hawley M E, Markiewicz D J, Spaepen F, and Barth EP (1999) Magnetic structure of deformation-induced shear bands in amorphous $\mathrm{Fe}_{80} \mathrm{~B}_{16} \mathrm{Si}_{4}$ observed by magnetic force microscopy. J. Appl. Phys. 85, 4415.

Bruck H A, Rosakis A J, and Johnson W L (1996) The dynamic compressive behavior of beryllium bearing bulk metallic glasses. $J$. Mater. Res. 11, 503-511.

Chang H J, Kim D H, Kim Y M, Kim Y J, and Chattopadhyay K (2006) On the origin of nanocrystals in the shear band in a quasicrystal forming bulk metallic glass $\mathrm{Ti}_{40} \mathrm{Zr}_{29} \mathrm{Cu}_{9} \mathrm{Ni}_{8} \mathrm{Be}_{14}$. Scripta Mater. 55, 509-512.

Cheng Y Q and Ma E (2009) Configurational dependence of elastic modulus of metallic glass. Phys. Rev. B 80, 064104.

Cheng Y Q, Han Z, Li Y, and Ma E (2009) Cold versus hot shear banding in bulk metallic glass. Phys. Rev. B 80, 134115.

Ding J, Patinet S, Falk M L, Cheng Y Q, and Ma E (2014) Soft spots and their structural signature in a metallic glass. Proc. Natl. Acad. Sci. US A 111, 14052-14056.

Gibert C J, Ager J W, Schroeder V, Ritchie R O, Lloyd J P, and Graham J R (1999) Light emission during fracture of a Zr-Ti-Ni-Cu-Be bulk metallic glass. Appl. Phys. Lett. 74, 3809-3811.

Greer A L, Cheng Y Q, and Ma E (2013) Shear bands in metallic glasses. Mater. Sci. Eng. R 74, 71-132.

Gu X, Livi K J T, and Hufnagel T C (2003) Structure of shear bands in Zirconium-based metallic glasses observed by transmission election microscopy. Mater. Res. Soc. Proc. 754, CC7.9.1.-CC7.9.6.

Guo H, Yan P F, Wang Y B, Tan J, Zhang Z F, Sui M L, and Ma E (2007) Tensile ductility and necking of metallic glass. Nature Mater. 6, 735739.

Han Z, Wu W F, Li Y, Wei Y J, and Gao H J (2009) An instability index of shear band for plasticity in metallic glasses. Acta Mater. 57, 13671372.

Hofmann D C (2010) Shape memory bulk metallic glass composites. Science 329, 1294-1295.

Lee M H, Lee K S, Das J, Thomas J, Kühn U, and Eckert J (2010) Improved 
plasticity of bulk metallic glasses upon cold rolling. Scripta Mater. 62, 678-681.

Lewandowski J J and Greer A L (2006) Temperature rise at shear bands in metallic glasses. Nature Mater. 5, 15-18.

Liu C T, Heatherly L, Easton D S, Carmichael C A, Schneibel J H, Chen C H, Wright J L, Yoo M H, Horton J A, and Inoue A (1998) Test environments and mechanical properties of Zr-base bulk amorphous alloys. Metall. Mater. Trans. A 29, 1811-1820.

Lu J, Ravichandran G, Johnson WL (2003) Deformation behavior of the $\mathrm{Zr}_{41.2} \mathrm{Ti}_{13.8} \mathrm{Cu}_{12.5} \mathrm{Ni}_{10} \mathrm{Be}_{22.5}$ bulk metallic glass over a wide range of strain-rates and temperatures. Acta. Mater. 51, 3429-3443.

Masumoto T and Maddin R (1975) Structural stability and mechanical properties of amorphous metals. Mater. Sci. Eng. 19, 1-24.

Megusar J, Argon A S, and Grant N J (1979) Plastic flow and fracture in Pd80Si20 near Tg. Mater. Sci. Eng. 38, 63-72.

Pauly S, Gorantla S, Wang G, Kühn U, Eckert J (2010a) Transformationmediated ductility in CuZr-based bulk metallic glasses. Nature Mater. 9, 473-477.

Pauly S, Liu G, Gorantla S, Wang G, Kühn U, Kim DH, Eckert J (2010b) Criteria for tensile plasticity in $\mathrm{Cu}-\mathrm{Zr}$-Al bulk metallic glasses. Acta. Mater. 58, 4883-4890.

Reynolds 0 (1885) On the dilatancy of media composed of rigid particles in contact. Phil. Mag. 20, 469-481.

Schall P, Weitz D A, and Spaepen F (2007) Structural rearrangements that govern flow in colloidal glasses. Science 318, 1895-1899.

Schuh C A, Hufnagel T C, and Ramamurty U (2007) Mechanical behavior of amorphous alloys. Acta Mater. 55, 4067-4109.
Schuh C A, Lund A C, and Nieh T G (2004) New regime of homogeneous flow in the deformation map of metallic glasses: elevated temperature nanoindentation experiments and mechanistic modeling. Acta Mater. 52, 5879-5891.

Shan Z W, Li J, Cheng Y Q, Minor A M, Syed Asif S A, Warren O L, and Ma $E$ (2008) Plastic flow and failure resistance of metallic glass: Insight from in situ compression of nanopillars. Phys. Rev. B 77, 155419.

Song S X and Nieh T G (2009) Flow serration and shear-band viscosity during inhomogeneous deformation of a $\mathrm{Zr}$-based bulk metallic glass. Intermetallics 17, 762-767.

Song S X, Bei H, Wadsworth J, and Nieh T G (2008) Flow serration in a Zr-based bulk metallic glass in compression at low strain rates. Intermetallics 16, 813-818.

Spaepen F (1977) A microscopic mechanism for steady state inhomogeneous flow in metallic glasses. Acta Mater. 25, 407-415.

Spaepen F (2006) Metallic glasses: Must shear bands be hot? Nature Mater. 5, 7-8.

Wu Y, Xiao Y, Chen G, Liu C T, and Lu Z P (2010) Bulk Metallic Glass Composites with Transformation-Mediated Work-Hardening and Ductility. Adv. Mater. 22, 2770-2773.

Wu Y, Zhou D Q, Song W L, Wnag H, Zhang Z Y, Ma D, Wang X L, and Lu Z P (2012) Ductilizing bulk metallic glass composite by tailoring stacking fault energy. Phys. Rev. Lett. 109, 245506.

Yang B, Liaw P K, Wang G, Morrison M, Liu C T, Buchanan R A, and Yokoyama Y (2004) In-situ thermographic observation of mechanical damage in bulk-metallic glasses during fatigue and tensile experiments. Intermetallics 2, 1265-1274.

http://faculty.virginia.edu/teamhowe/files/EMFacility.html 